\title{
Structural Insights into Catalytic Versatility of the Flavin-dependent Hydroxylase (HpaB) from Escherichia coli
}

John P. Rose ${ }^{a}$, Xiaolin Shen ${ }^{b, c}$, Dayong Zhou ${ }^{a}$, Yuheng Lin ${ }^{d}$, Jia Wang ${ }^{b, c}$, Shuaihua Gao ${ }^{b, c}$, Palani Kandavelu $^{a}$, Hua Zhang ${ }^{a}$, Ruihua Zhang ${ }^{d}$, Bi-Cheng Wang ${ }^{a}$, Qipeng Yuan ${ }^{b, c}$, Yajun Yan ${ }^{d}$

aDepartment of Biochemistry \& Molecular Biology, The University of Georgia, Athens, Georgia 30602, USA iprose@uga.edu, dayzhou@bcl4.bmb.uga.edu, palani@anl.gov, hzhang123@yahoo.com, bcwang@uga.edu

${ }^{b} B e i j i n g$ Advanced Innovation Center for Soft Matter Science and Engineering, Beijing University of Chemical Technology, Beijing 100029, China shenxl@mail.buct.edu.cn, wangiia@mail.buct.edu.cn, shuaihuagao@yahoo.com, yuanqp@mail.buct.edu.cn

'State Key Laboratory of Chemical Resource Engineering, Beijing University of Chemical Technology, Beijing 100029, China shenxl@mail.buct.edu.cn, wangjia@mail.buct.edu.cn, yuanqp@mail.buct.edu.cn

'School of Chemical, Materials and Biomedical Engineering, College of Engineering, The University of Georgia, Athens, GA 30602, USA yuheng.lin@biotecera.com, rz36661@uga.edu, yajunyan@uga.edu

The enzyme 4-Hydroxyphenylacetate 3-hydroxylase (EcHpaB) from Escherichia coli is capable of efficient ortho-hydroxylation of a wide range of phenolic compounds and demonstrates great potential for broad chemoenzymatic applications. To understand the structural and mechanistic basis of its catalytic versatility, we elucidated the crystal structure of $\mathrm{EcHpaB}$ by X-ray crystallography [1], which revealed a unique loop structure covering the active site. We further carried out mutagenesis studies of this loop to probe its role in substrate specificity and catalytic activity. Our results not only showed the loop has great plasticity and strong tolerance towards extensive mutagenesis, but also suggested this flexible loop that enables the entrance and stable binding of substrates into the active site is the key factor to the enzyme catalytic versatility. These findings laid the groundwork for editing the loop sequence to effect loop structure that led to the generation of EcHpaB loop mutants with improved enzymatic performance against larger nonnatural substrates. Details of the analysis will be presented.

\section{References}

Shen, et al. "Structural Insights into Catalytic Versatility of the Flavin-dependent Hydroxylase (HpaB) from Escherichia coli." Scientific reports 9.1 (2019): 7087. 